\title{
Finding Pull Directions for Interference-free Mould Openings
}

\author{
Alan C. Lin ${ }^{1, a}$ and Mohammad Effendi ${ }^{1}$ \\ ${ }^{1}$ Department of Mechanical Engineering, National Taiwan University of Science and Technology, 43, Section 4, \\ Keelung Road, Taipei 10607, Taiwan.
}

\begin{abstract}
This paper proposes an approach to determining pull directions of an undercut region using the visibility map algorithm. A number of pull direction candidates are firstly generated and projected onto a spherical surface using a regular placement algorithm. The normal directions of undercut faces are then extracted and used to eliminate unwanted pull direction candidates. A group of pull directions are finally selected and used to design a side core mechanism in the design of an injection mould. Furthermore, one point at the center of the group is chosen to represent the pull direction of the undercut region.
\end{abstract}

\section{Introduction}

Figure 1(a) shows a typical cell phone component with holes and undercut areas distributed in various locations. To manufacture the component using injection moulding, several sliders and lifters with appropriate pull directions are needed, as shown in Figure 1(b). In the field of mould manufacturing, the determination of pull directions is critical to design sliders and lifters so that the mould can be opened without any interference [1]. A number of studies have been proposed regarding this issue. For example, Nee et al. [1] proposed a method based on geometrical characteristic and topological relationship, where the pull direction is obtained from normal directions of undercut faces. However, this method fails for cases with irregular faces. Lin et al. [2, 3] use Visibility Map (V-map) algorithms to determine the pull direction of an undercut region based on the STL format. However, the interference between the core and moulding parts shows that their algorithm should be improved. Based on attributes and nodes in an Extended Face Adjacency Attribute Graph (EFAAG) algorithm, Jian and Guoxiang [4] proposed a method for recognizing undercut regions. The V-map is used in their method to find pull directions of an undercut. Chen and Chou [5] introduced Augmented Visibility Map (AVM) to minimize the number of undercut regions for pockets. Chen et al. [6] proposed an algorithm to determine pull directions based on V-map using a Gaussian Mapping method. The purpose of their research was to minimize the number of cores based on the Pair of Parting Direction (PPD) algorithm. The pull direction is calculated with the Maximally Covered Point (MCP) algorithm. Liu and Ramani [7] proposed an exact visibility map method based on facet triangle meshing. Each facet of the V-map is projected onto a spherical surface, and then the intersection between all of it is selected as undercut pull direction.

\footnotetext{
${ }^{\text {a }}$ Corresponding author: alin@mail.ntust.edu.tw
} 


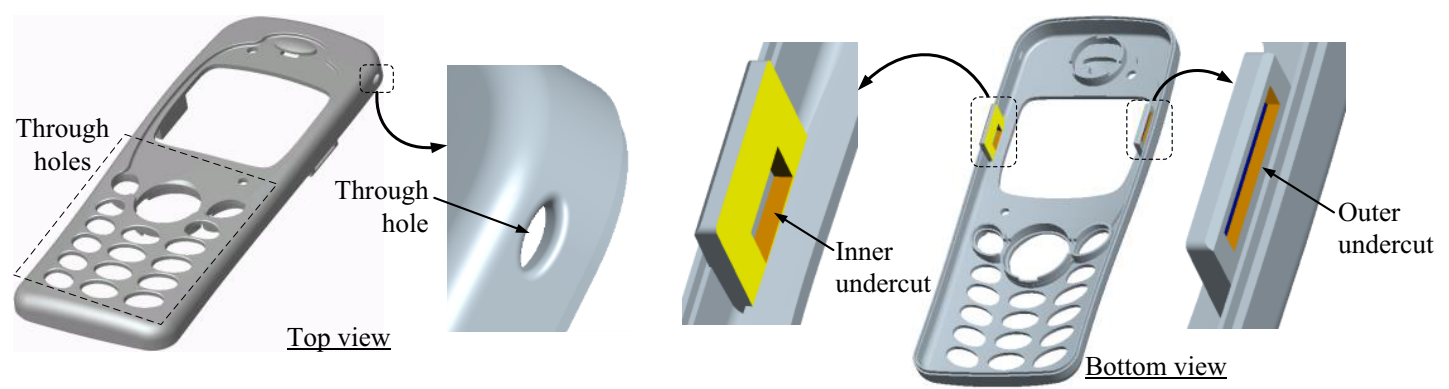

(a)

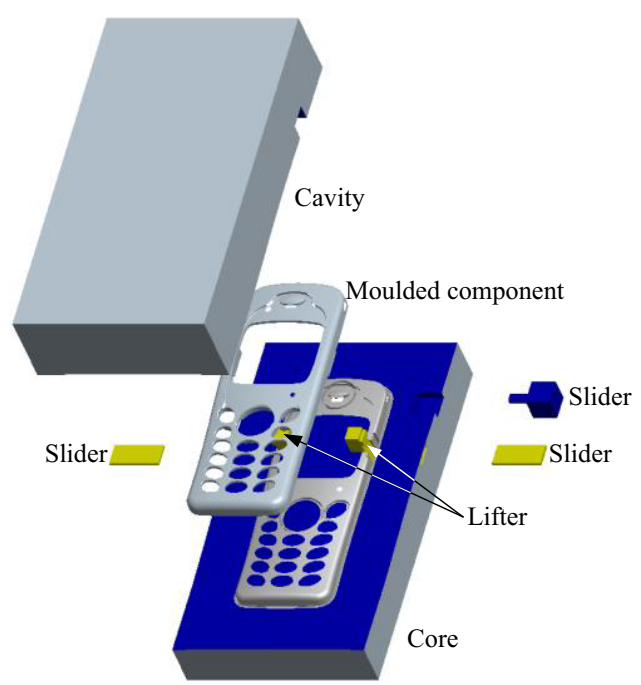

(b)

Figure 1. Normal directions of $(a)$ an irregular face and $(b)$ a regular face.

Most of the above mentioned approaches use regular faces and work only for limited cases. To overcome this problem, this paper proposes a method to determine pull directions of an undercut region with both regular and irregular faces.

\section{Determination of Pull Directions}

In our proposed method, a number of pull direction candidates are first determined and plotted onto a spherical surface using a regular placement algorithm as V-map representation. Then, the normal direction of both regular and irregular faces are extracted to create a 3D circle line. The V-map region is then built based on the 3D circle line and the normal direction, and used to eliminate unwanted pull direction candidates. A group of pull direction candidates of an undercut is finally selected, as the intersection of all V-maps through the Boolean operation. A center point of the group is then used as the pull direction for the undercut region. Figure 2 shows the proposed algorithm, where all the steps are described in the following sections.

\subsection{Calculation of Normal Directions}

Faces of an undercut have been classified into regular and irregular faces. An irregular face is a common face for complex manufacturing products such as turbine blades, cell phones, art products based on 3D printing, etc. For such faces the normal direction is perpendicular to the tangent plane at the selected point. A mesh in $x$-, $y$ - and $z$ - coordinates is then set and used as a sampling tool to obtain 


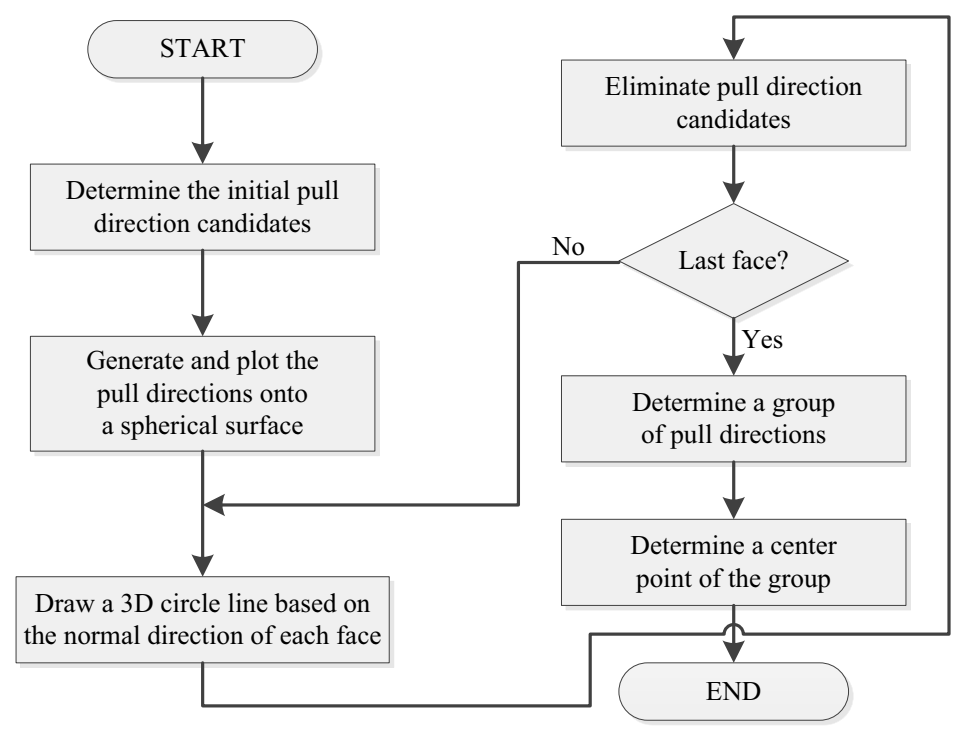

Figure 2. Flowchart of the proposed method.

normal direction, which depends on the coordinates of mesh. For a regular face, e.g. a plane, the normal directions are perpendicular to its plane at selected points. Figure 3 highlights an illustration of the normal directions for both of regular and irregular face. To overcome this problem, the related program is employed using C\# code and OpenNX API (Application Program Interface).

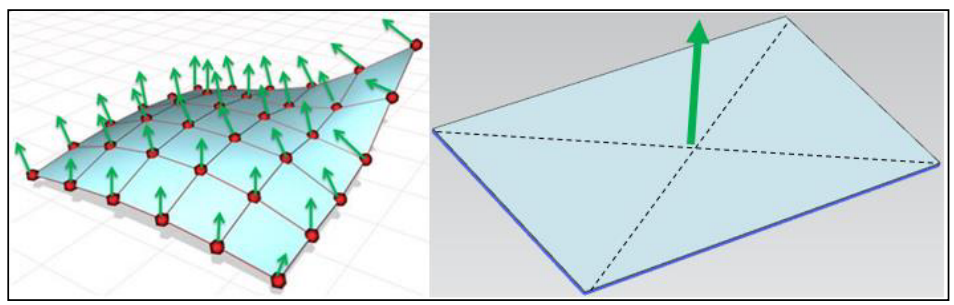

(a)

(b)

Figure 3. Normal directions of $(a)$ an irregular face and $(b)$ a regular face.

In OpenNX, the AskFaceData function is used to calculate the normal direction for a regular face. The AskFaceData, AskFaceParm, and AskFaceProps functions are used together to find normal direction for irregular faces, as shown in the Figure 4. The normal directions of both regular and irregular faces, are then collected and used to select pull direction candidates for an undercut based on the V-map principle.

\subsection{Generation and Projection of Pull Direction Candidates}

Deserno [8] proposed two methods to generate and distribute points onto a spherical surface. A distributed point denotes a pull direction candidate of an undercut. The first method called the "random placement method", randomly plots the distributed points onto a spherical surface. Fluctuation and overlapping of distributed points show the weakness of this method, therefore the points are not evenly distributed, as can be seen in the area 1 and 2 in Figure 5(a). The second method called the "regular placement method", places each point from the other point at the same distance in two orthogonal directions, as can be seen in the Figure 5(b). Using this method, all of the determined points can be evenly distributed onto the spherical surface. Although the second method shows an emerging partial crystallinity problem, our preliminary results show that it gives better performance. We therefore choose the second method. 


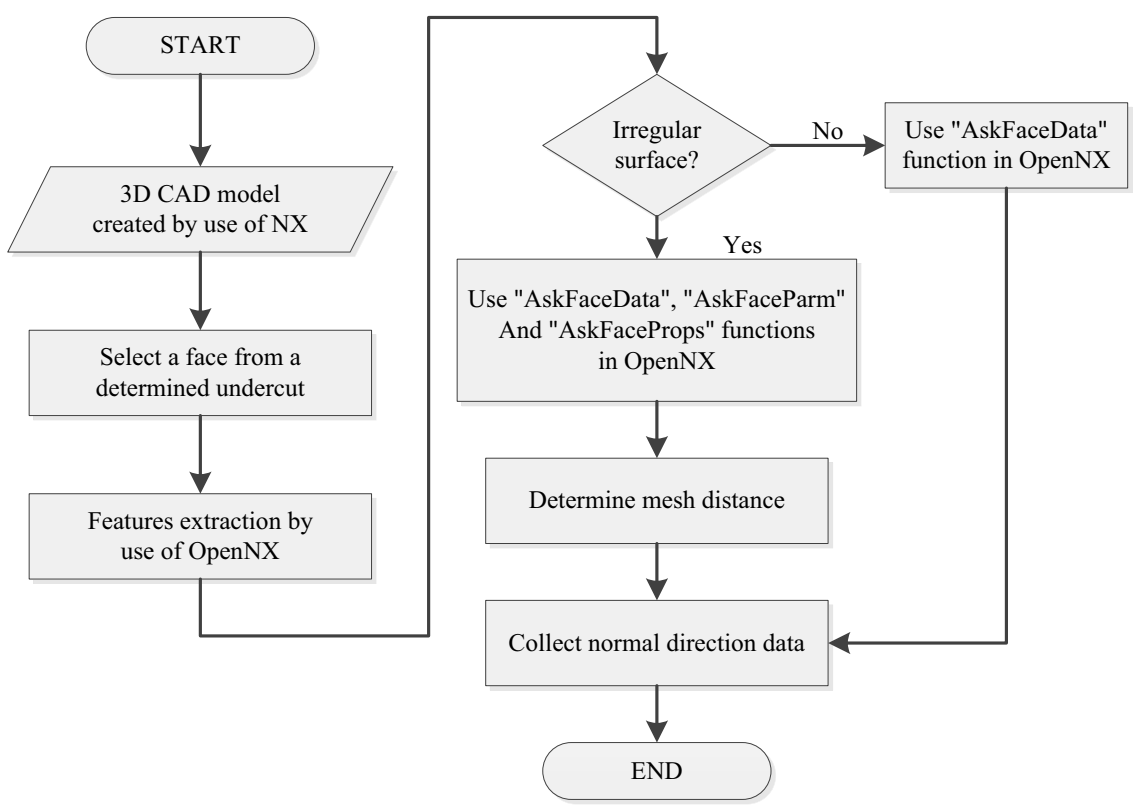

Figure 4. Flowchart to calculate normal directions of undercut's faces.

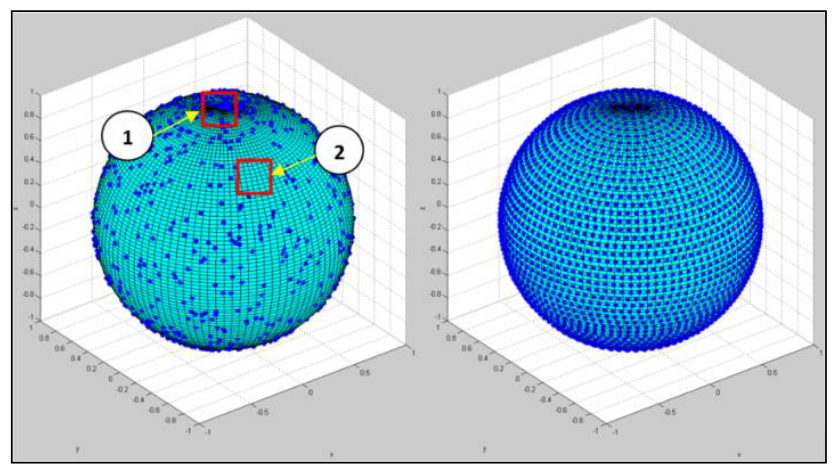

(a)

(b)

Figure 5. Projection of pull direction candidates using $(a)$ random placement, and $(b)$ regular placement method.

\subsection{Visibility Map of an Undercut Region}

To illustrate the basic concept of the proposed method, a simple undercut is considered for demonstration. Figure 6 shows an undercut with 5 different regular faces, where the normal direction of each face can be seen in Table 1. Figure 7(a) highlights the blue points, which shows the pull direction candidates of an undercut region. The red line shows the $3 \mathrm{D}$ circle location, and the yellow point shows the normal direction of a face. Both of the red line and the yellow point represent the location of the face's V-map region.

The normal direction of a face is used as a reference to create a $3 \mathrm{D}$ circle line. It represents $\mathrm{V}$-map which is used to eliminate unwanted pull direction candidates. The distance between the $3 \mathrm{D}$ circle line and the normal direction point is then calculated and used as a threshold to perform it. The distance between each drawing candidate and the normal direction point is calculated for the next step. If the distance is greater than the threshold, then the candidate will be removed, otherwise it will be kept for the next step. Thereby, the number of pull direction candidates will be gradually reduced, and the center point of leftover candidates is chosen as the undercut's pull direction. 


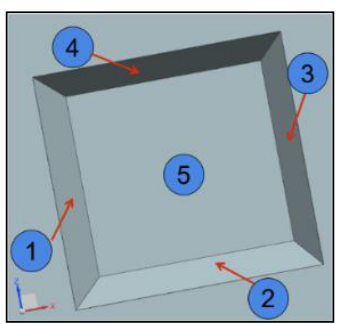

Figure 6. Example of undercut with 5 regular faces.

Table 1. Normal directions of an undercut faces in Figure 6.

\begin{tabular}{|c|c|c|c|}
\hline Face No. & $\boldsymbol{X}$ normal direction & $\boldsymbol{Y}$ normal direction & $\boldsymbol{Z}$ normal direction \\
\hline 1 & 0.985 & -0.174 & 0 \\
\hline 2 & 0 & -0.174 & 0.985 \\
\hline 3 & -0.985 & -0.174 & 0 \\
\hline 4 & 0 & -0.174 & -0.985 \\
\hline 5 & 0 & -1 & 0 \\
\hline
\end{tabular}

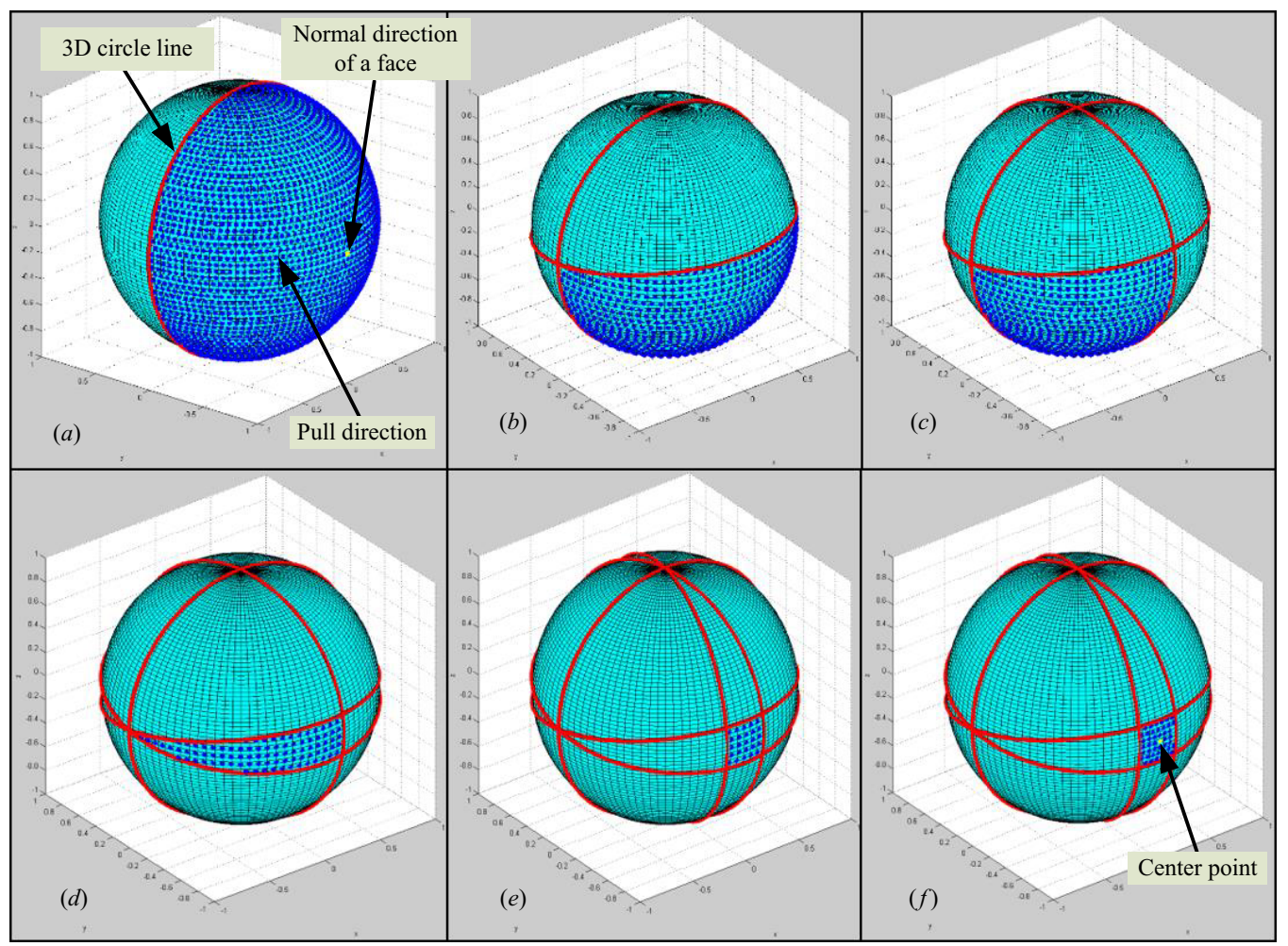

Figure 7. Step by step illustration of the proposed method.

In the demonstration, five thousand pull direction candidates are eliminated with the V-map of the first face, leaving 2,498 candidates as can be seen in the Figure 7(a). Similarly, Figure 7(b) shows the 1,272 pull direction candidates after using the V-map of the second face. Furthermore, the V-map of the third face is used to eliminate the pull direction into 658 candidates as can be seen in the Figure $7(c)$. In the next step, the V-map of the fourth face is used to reduce the pull direction into 163 candidates as we see in the Figure $7(d)$. 
After elimination by the V-map of the last face, 49 pull directions are finally obtained as shown in Figure 7(e). These points show an intersection region of all face's V-maps based on a Boolean operation, which is formulated by the following equation:

$$
V m a p=\bigcap_{i=1}^{m} \operatorname{Vmap}_{i}
$$

where $m$ is the total number of undercut faces. Center point $(0.0126-0.99990)$ is chosen to represent 49 directions, as shown in Figure 7(f).

\section{System Implementation}

Figure 8(a) shows an undercut with 13 different irregular faces, and is used to illustrate the applicability and advantages of the proposed method. The mesh size is set at $4 \mathrm{~mm} \times 4 \mathrm{~mm} \times 4 \mathrm{~mm}$, and the total number of normal direction is 103. The initial number of undercut pull direction candidates is set at 10,000 points, which are generated by a regular placement method. After elimination, the total number of drawing candidates is 839 . Finally, the point [0.1542 $-0.03520 .9874]$ is chosen as the center point to represent 839 candidates as can be seen in the Figure $8(b)$.

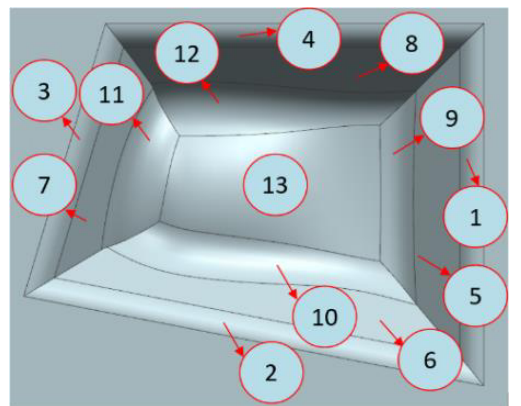

(a)

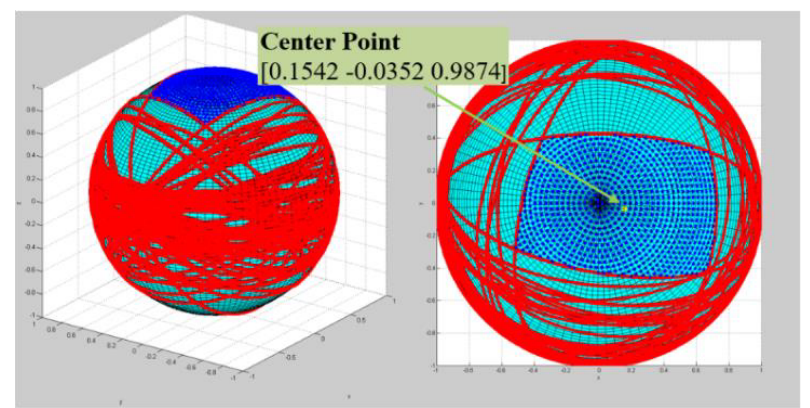

(b)

Figure 8. (a) Undercut with irregular surfaces, (b) pull directions (blue) bounded by 3D circle lines (red).

\section{Conclusions}

An algorithm for automatic determination of an undercut pull direction for both regular and irregular faces is presented in this paper based on the V-map method. The pull direction candidates are generated by a random placement method, and plotted onto a spherical surface. The normal directions of undercut faces are extracted to create $\mathrm{V}$-map regions, and used to eliminate unwanted pull direction candidates. Eventually, a group of drawing candidates is selected, and a point in the center of the group is chosen to represent the pull direction for the undercut region. The pull direction becomes a vital element in the design of an injection mould.

\section{References}

1. A. Nee, M. Fu, J. Fuh, K. Lee, Y. Zhang, Ann. of the CIRP, 46 (1997).

2. A. Lin, A. Son, App. Mech. and Mat., 275-277, 2606-2610 (2013).

3. N. Quang, A. Lin, App. Mech. and Mat., 275-277, 2635-2639 (2013).

4. S. Jian, S. Guoqiang, ICSE, 1468-1471 (2010).

5. L. Chen, S. Chou, Jour. of Man. Sys, 14 (1995).

6. L. Chen, S. Chou, T. Woo, Comp.-Aid. Des., 25 (1993).

7. M. Liu, K. Ramani, Sol. and Phys. Mod. Symp., 367-372 ( 2007).

8. M. Deserno, Technical Report, Department of Chemistry and Biochemistry, UCLA, USA (2004). 